\title{
Research on Vibration Suppression of the Long Flexible Arm Based on Three Tilt Sensors
}

\author{
Haiyong Jiang $\mathbb{D},{ }^{1,2}$ Wenguang Jiang $\left(\mathbb{D},{ }^{1}\right.$ Yazhou Xing, ${ }^{2} \mathrm{Na} \mathrm{Li},{ }^{2}$ and Xin Yang ${ }^{2}$ \\ ${ }^{1}$ Hebei Innovation Center for Equipment Lightweight Design and Manufacturing, School of Mechanical Engineering, \\ Yanshan University, Qinhuangdao 066004, Hebei, China \\ ${ }^{2}$ Mechanical Engineering Department, College of Electrical and Mechanical Engineering, Hebei Agricultural University, \\ Baoding 071001, Hebei, China \\ Correspondence should be addressed to Wenguang Jiang; wgj@ysu.edu.cn
}

Received 13 August 2021; Revised 8 September 2021; Accepted 17 September 2021; Published 12 October 2021

Academic Editor: Sang-Bing Tsai

Copyright ( 2021 Haiyong Jiang et al. This is an open access article distributed under the Creative Commons Attribution License, which permits unrestricted use, distribution, and reproduction in any medium, provided the original work is properly cited.

\begin{abstract}
In this research, a new method based on the equivalence of modal characteristics, differential flatness (DF), and active disturbance rejection control (ADRC) is proposed for the stabilization control of the long flexible arm (LFA). There are two major problems in the system of the LFA. The first problem is that the LFA is very prone to the multiple-mode coupling, while the control systems need as few sensors as possible. Another problem is that the structure of the LFA in practice is often complex and subject to various disturbances. Therefore, in this paper, the equivalent multirigid body dynamic model of a LFA is derived from the modal information of the equivalent rigid body model of the prototype. Then, the output values of the three tilt sensors are synthesized into an output based on the DF method. Finally, the effectiveness of the proposed method is verified through physical experiments. Compared with PID, the proposed method has shorter settling time. The LFA can be restored within 7 seconds under the ADRC, while it needs 90 seconds or more to calm down without the control.
\end{abstract}

\section{Introduction}

The application of the long arm structure in industrial and agricultural production is more and more extensive with the rapid development of the mechanical industry [1-4]. In the field of agricultural engineering, pruning and harvesting devices for high canopy are mostly made of lightweight materials to reduce costs and increase mobility. However, this results in low structural rigidity. In addition, with the improvement of energysaving awareness and high maneuverability design requirements, lightweight design is the general trend. The vibration problem caused by significant flexibility brings troubles to the agricultural and engineering equipment. This poses a great challenge to the stability of long flexible arm structures.

LFA is subject to many disturbances such as working load, bumping, or wind. It takes too much time to stabilize by means of self-damping, which will seriously reduce working efficiency and even lead to serious accidents. Moreover, in the arm structure, the driving action point is located at the lower part of the arm, which leads to the multimode coexistence under the action of variable amplitude force. Vibration not only limits the working efficiency of the equipment but also becomes a serious hidden danger in the production process.

Several studies use the continuum model analysis method for dynamic modeling and response analysis of some beam structures. Weldegiorgis et al. [5] derived the model for a 0.3 -metre-long cantilever beam using the system identification technique and then used the piezoelectric patches as the sensor and actuator to suppress the vibration of the beam. However, this method is difficult to be concise and effective due to the complex structure, uncertain contact condition, complex interference, and other factors in the real LFA. He et al. [6, 7] modeled the flexible arm and end-effector as a homogeneous cantilever beam and a concentrated mass and used the neural network control method in conjunction with the lumped spring-mass model to realize the stability of the flexible beam on the Quanser platform. 
It is a practical goal to give a simple and quick way to get the dynamic model for the lower fundamental frequency flexible arm subjected to gravity and impact. In recent years, the pseudo-rigid body model (PRBM) method is widely used in the research of flexible body modeling $[8,9]$. The pseudorigid body method has achieved remarkable achievements in the exact equivalent deformation of large deformation structures with flexible bodies [10]. We use the PRBM idea to carry out the dynamic equivalence in order to consider the complex and large-scale interference factors. Guided by the PRBM method, the rigid body equivalent of the flexible beam can be obtained by directly using the measured data as the output. Then, the LFA can be simplified by the equivalence of static deformation and the first two modes. The whole LFA is equivalent to three rigid segments, and the equivalent dynamic model including the single torque input and three-angle output is constructed to become an underactuated system.

Ramírez-Neria and coresearchers solved several kinds of underactuated mechanical systems successfully by using the differential flattening method combined with the ADRC or generalized proportional integral observer. Successful cases include the rotary inverted pendulum [11], wheel pendulum system, and underactuated mobile manipulators [12, 13], which have fully demonstrated the advantages of combining the dimension reduction processing of the differential flattening method with the active disturbance rejection compensation for internal and external disturbances. SiraRamirez [14] said that the flat output function is rather elaborate and complicated to give. For the convenience of engineering applications, we need to explore a relatively more direct way to obtain flat output variables.

As an important progress in the field of control engineering in the past two decades, ADRC is gradually understood and recognized by the industry. A large number of studies have shown that ADRC has good performance on complex linear or nonlinear systems with uncertainties [15-17]. In order to deal with the uncertain internal or external disturbances and the higher-order ones caused by differential flattening, ADRC method can deal with these difficulties effectively and skillfully [18].

The contributions of this manuscript are as follows:

(i) The modal equivalent method of transforming the LFA into the rigid body model is built.

(ii) A differential flat output for the three-bar twotorsion-spring mechanism is constructed based on the differential flat principle, and a single-input single-output system model in the form of differential equations is established.

(iii) Taking the differential flat output as the system output, PID and ADRC controllers are used to suppress the vibration under impact disturbance, respectively. The experiment is carried out on the long arm experimental device.

The rest of this paper is organized as follows: in Section 2, the modeling method of the equivalent rigid body model for the cantilever section of the LFA is described. In Section 3, the equivalent dynamic model analysis of three rods and two springs is carried out. Section 4 discusses the differential flatness method for the single-input multiple-output system. In Section 5, a single differential flat output and single torque input stabilization system with the linear active disturbance rejected control method is studied. In Section 6, the effectiveness of this method is verified by experiments, and the manuscript is concluded in Section 7.

\section{Equivalent Model of the LFA}

According to the principle of the mode superposition method, the lower modes are the main components of the dynamic response. The internal structure of the LFA is often so complex that it is difficult to build an accurate kinetic model according to the mechanism. In order to construct an algorithm with wide applicability, the LFA on the left side in Figure 1 is equivalent to the plane multirigid body and spring system on the right side according to the modal information. When the LFA is disturbed and shakes, the first and second modes are the most significant in the dynamic response process, so the LFA is regarded as a planar hinge mechanism composed of three rods and two torsion springs, which is shown in Figure 1. The multirigid body dynamic method is used to get the equivalent dynamic response of the continuum arm approximately. The symbols are defined as follows: $\theta_{0}$ is the expected elevation of the flexible long arm, $\theta_{1}, \theta_{2}$, and $\theta_{3}$ are the elevations of the equivalent rods relative to the horizontal plane, and $l_{i}, m_{i}, I_{c i}$, and $k_{i}$ represent the rod length, mass, moment of inertia according to the mass centers, and torsion spring stiffness of the $i$ th rod in the equivalent mechanism, respectively. In order to simplify the derivation process, the torque at the bottom of the first rod is used to represent the force $F_{d}$ acting at point $O$ '.

The tilt sensor based on MEMS technology has been very cheap, even the price of the vibration detection type has been reduced to a hundred dollars, so it is used to collect kinematic information of the LFA in the process of vibration. In order to obtain more valuable dynamic information, we use the method of arranging tilt sensors at the root, middle, and top of the LFA to establish the feedback control system. The part between the fixed hinge point $O$ of the LFA and the action point $O^{\prime}$ of the luffing support is usually stronger as the support part. Because the flexibility of this section is weak and ignored, it is directly represented as a rigid body. So, the segment $O O^{\prime}$ is taken as the first rod of the equivalent multirigid-body and spring (MRB\&S) model.

Then, we need to determine the installation position on the LFA for every tilt sensor. Tilt sensor 1 can be installed at the root of the LFA at will, while for tilt sensor 3, it can be seen that the vibration of rod 3 can be obtained more significantly when it is installed at the end. The key task in this stage is to determine the installation location of tilt sensor 2, that is, to determine the value of $l_{k 1}$. Furthermore, we need to determine the mass and length of each rod in the equivalent model and the stiffness coefficient of the torsion spring. 


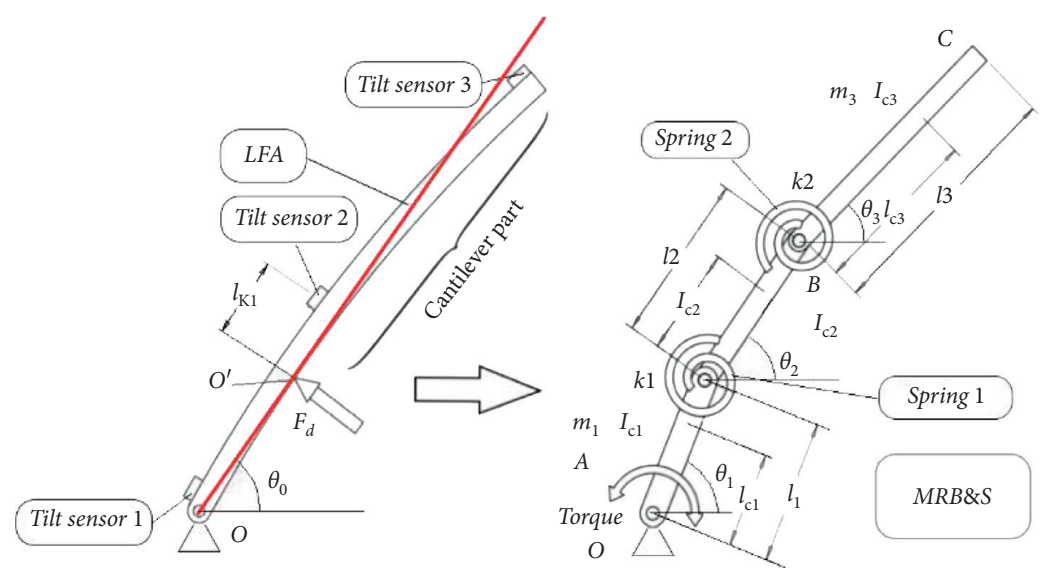

FIgURE 1: The LFA is equivalent to a MRBS system.

The specific process is as follows: firstly, the length of two equivalent rigid bars and the sensor installation position on the LFA are determined by parameter matching according to the second-order mode shape of the continuous arm. The mass of two rigid bars and the stiffness coefficients of two equivalent torsion springs are unknown. Then, the static deformation of the cantilever section, the static moment at the root, and the mode shapes of the first two modes are used to determine the four undetermined coefficients.

2.1. Dynamic Model of the Two-Rod and Two-Spring Mechanism. We use the Euler-Lagrange formulism to derive the model of the system. The kinetic energy $T_{i}$ and potential energy $V_{i}$ of rod 2 and $\operatorname{rod} 3$ are

$$
\left\{\begin{array}{l}
T_{2}=\frac{1}{2}\left(I_{c 2}+m_{2} l_{c 2}^{2}\right) \dot{\theta}_{2}^{2}, \\
V_{2}=m_{2} g l_{c 2} \sin \theta_{2}, \\
T_{3}=\frac{1}{2} m_{3}\left[l_{2}^{2} \dot{\theta}_{2}^{2}+l_{c 3}^{2} \dot{\theta}_{3}^{2}+2 l_{2} l_{c 3} \dot{\theta}_{2} \dot{\theta}_{3} \cos \left(\theta_{2}-\theta_{3}\right)\right]+\frac{1}{2} I_{c 3} \dot{\theta}_{3}^{2}, \\
V_{3}=m_{3} g\left(l_{2} \sin \theta_{2}+l_{c 3} \sin \theta_{3}\right) .
\end{array}\right.
$$

The elastic potential energy of two equivalent torsion springs is

$$
V_{k}=\frac{1}{2}\left[k_{1}\left(\theta_{1}-\theta_{2}\right)^{2}+k_{2}\left(\theta_{2}-\theta_{3}\right)^{2}\right] .
$$

$$
\begin{aligned}
L= & \frac{1}{2} m_{3}\left[l_{2}^{2} \dot{\theta}_{2}^{2}+l_{c 3}^{2} \dot{\theta}_{3}^{2}+2 l_{2} l_{c 3} \dot{\theta}_{2} \dot{\theta}_{3} \cos \left(\theta_{2}-\theta_{3}\right)\right]-\frac{1}{2}\left[k_{1}\left(\theta_{1}-\theta_{2}\right)^{2}+k_{2}\left(\theta_{2}-\theta_{3}\right)^{2}\right] \\
& +\frac{1}{2}\left(I_{c 2}+m_{2} l_{c 2}^{2}\right) \dot{\theta}_{2}^{2}+\frac{1}{2} I_{c 3} \dot{\theta}_{3}^{2}-m_{2} g l_{c 2} \sin \theta_{2}-m_{3} g\left(l_{2} \sin \theta_{2}+l_{c 3} \sin \theta_{3}\right) .
\end{aligned}
$$


According to the principle of Lagrange dynamics,

$$
\frac{\mathrm{d}}{\mathrm{d} t}\left(\frac{\partial T}{\partial \dot{q}_{j}}\right)-\frac{\partial T}{\partial q_{j}}=\tau_{j}
$$

$$
\left\{\begin{array}{l}
\left(m_{2} l_{c 2}^{2}+m_{3} l_{2}^{2}+I_{c 2}\right) \ddot{\theta}_{2}+m_{3} l_{2} l_{c 3}\left[\cos \left(\theta_{2}-\theta_{3}\right) \ddot{\theta}_{3}+\sin \left(\theta_{2}-\theta_{3}\right) \dot{\theta}_{3}^{2}\right]+\left(m_{2} l_{c 2}+m_{3} l_{2}\right) g \cos \theta_{2}-k_{1}\left(\theta_{1}-\theta_{2}\right)+k_{2}\left(\theta_{2}-\theta_{3}\right)=0 \\
\left(m_{2} l_{c 3}^{2}+I_{c 2}\right) \ddot{\theta}_{3}+m_{3} l_{2} l_{c 3}\left[\cos \left(\theta_{2}-\theta_{3}\right) \ddot{\theta}_{2}-\sin \left(\theta_{2}-\theta_{3}\right) \dot{\theta}_{2}^{2}\right]+m_{3} g l_{c 3} \cos \theta_{3}-k_{2}\left(\theta_{2}-\theta_{3}\right)=0 .
\end{array}\right.
$$

Let $\theta_{i 0}$ be the initial value of the angle when the $i$ th rod is in the static state, and $\theta_{i}$ of each rod occurs near its respective static values, and the angle increment $\theta_{\delta i}$ deviating from the equilibrium position is small. It means that $\theta_{\delta i}=\theta_{i}-\theta_{i 0}$.
The differences between the elevation angles of the adjacent rigid bars are small, generally not more than 0.09 radians in the equilibrium state. Using the small deformation assumption for linearization, the value of (6) is approximately determined.

$$
\left\{\begin{array}{l}
\theta_{1}-\theta_{2} \approx 0, \\
\theta_{2}-\theta_{3} \approx 0, \\
\cos \left(\theta_{1}-\theta_{2}\right) \approx 1, \\
\cos \left(\theta_{2}-\theta_{3}\right) \approx 1 \\
\sin \left(\theta_{1}-\theta_{2}\right) \approx \theta_{1}-\theta_{2}, \\
\sin \left(\theta_{2}-\theta_{3}\right) \approx \theta_{2}-\theta_{3}, \\
\dot{\theta}_{2}^{2} \approx 0, \dot{\theta}_{3}^{2} \approx 0 .
\end{array}\right.
$$

Then, equation (5) can be simplified as

$$
\left\{\begin{array}{l}
\left(m_{2} l_{c 2}^{2}+m_{3} l_{2}^{2}+I_{c 2}\right) \ddot{\theta}_{2}+m_{3} l_{2} l_{c 3} \ddot{\theta}_{3}+\left(k_{1}+k_{2}\right) \theta_{2}+\left(m_{2} l_{c 2}+m_{3} l_{2}\right) g \cos \theta_{2}-k_{1} \theta_{10}-k_{2} \theta_{3}=0 \\
\left(m_{3} l_{c 3}^{2}+I_{c 2}\right) \ddot{\theta}_{3}+m_{3} l_{2} l_{c 3} \ddot{\theta}_{2}+m_{3} g l_{c 3} \cos \theta_{3}-k_{2}\left(\theta_{2}-\theta_{3}\right)=0 .
\end{array}\right.
$$

2.2. Equivalent of Static Conditions. The static balance includes two aspects: one is the balance state of the static deformation of the cantilever section, and the other is the balance between the root bending moment and the moment caused by the mass of the arm. The static equilibrium deformation and modal shape information of the actual LFA can be obtained by experiment, analysis, or finite element calculation. In the state of static equilibrium, according to the balance relationship between the torsion of the equivalent springs and the gravity of the rigid rods, the following equation can be obtained:

$$
\left\{\begin{array}{l}
k_{1}\left(\theta_{10}-\theta_{20}\right)=\frac{1}{2} m_{2} g l_{2} \cos \theta_{20}+m_{3} g\left(l_{2} \cos \theta_{20}+\frac{1}{2} l_{3} \cos \theta_{30}\right), \\
k_{2}\left(\theta_{20}-\theta_{30}\right)=\frac{1}{2} m_{3} g l_{3} \cos \theta_{30} .
\end{array}\right.
$$


Here, the two expressions can be merged into the following:

$$
k_{1}\left(\theta_{10}-\theta_{20}\right)-k_{2}\left(\theta_{20}-\theta_{30}\right)=\left(\frac{1}{2} m_{2}+m_{3}\right) g l_{2} \cos \theta_{20} .
$$

According to the triangular function formulas, we take the following approximation:

$$
\cos \theta_{i} \approx \cos \theta_{i 0}-\sin \theta_{i 0} \cdot \theta_{\delta i} .
$$

Equation (7) can be simplified as (11) when the center of each rod is at the mass center.

$$
\mathbf{M}\left[\begin{array}{l}
\ddot{\theta}_{\delta 3} \\
\ddot{\theta}_{\delta 3}
\end{array}\right]+\mathbf{K}\left[\begin{array}{c}
\theta_{\delta 3} \\
\theta_{\delta 3}
\end{array}\right]=0
$$

where

$$
\begin{aligned}
& \left\{\begin{array}{l}
a=m_{3} l_{2} l_{3}, \\
b=\left(\frac{1}{3} m_{2}+m_{3}\right) l_{2}^{2}, \\
c=\left(\frac{1}{2} m_{2}+m_{3}\right) g l_{2} \sin \theta_{20}, \\
d=\frac{1}{4} m_{3} l_{3}^{2}+\frac{1}{12} m_{2} l_{2}^{2}, \\
e=\frac{1}{2} m_{3} g l_{3} \sin \theta_{30},
\end{array}\right. \\
& \mathbf{M}=\left[\begin{array}{ll}
a & b \\
d & a
\end{array}\right], \\
& \mathbf{K}=\left[\begin{array}{cc}
-k_{2} & k_{1}+k_{2}-c \\
k_{2}-e & -k_{2}
\end{array}\right] \text {. }
\end{aligned}
$$

2.3. Equivalent of Two Mode Shapes. The characteristic matrix of the undamped simple harmonic vibration of the mechanism composed of two rods and two springs can be expressed as

$$
\mathbf{B}=\mathbf{K}-\omega^{2} \mathbf{M}\left[\begin{array}{cc}
-k_{2}-a \omega^{2} & k_{1}+k_{2}-c-b \omega^{2} \\
k_{2}-e-d \omega^{2} & -k_{2}-a \omega^{2}
\end{array}\right] .
$$

For a specific mode of a certain order, ratios $R_{1}$ and $R_{2}$ of the two tilt sensors' values can also be obtained by experiments, physical operation, or finite element method. The mechanism with two rigid rods and two torsion springs can be equivalent to the extension of the LFA in the sense of dynamics. In addition to the equivalent natural angular frequency, it must also meet the equivalent mode shape. In the equivalent rigid body model, the natural angular frequency values of the corresponding modes are substituted into characteristic matrix $B$ in (13), and the adjoint matrix adj $\mathbf{B}_{\omega_{(i 0)}}$ is obtained. In each adjoint matrix shown in (14), any row vector of the matrix can be used as the mode vector expressed by angle values. Corresponding to the LFA in practice, ratios $R_{1}$ and $R_{2}$ of the two amplitudes corresponding to the position of tilt sensor 2 and tilt sensor 3 in the first two modes can be obtained by modal analysis or modal experiment.

$$
\operatorname{adj} \mathbf{B}_{\left(\omega_{i 0}\right)}=\left[\begin{array}{cc}
-k_{2}-a \omega_{i 0}^{2} & -k_{1}-k_{2}+c+b \omega_{i 0}^{2} \\
-k_{2}+e+d \omega_{i 0}^{2} & -k_{2}-a \omega_{i 0}^{2}
\end{array}\right] .
$$

Two equivalent equilibria (15) can be obtained by making the ratio of the row vector elements in the equivalent mode shape and the corresponding mode angle ratio of the cantilever section of the LFA.

$$
\left\{\begin{array}{l}
k_{2}+a \omega_{10}^{2}=\left(k_{1}+k_{2}-c-b \omega_{10}^{2}\right) R_{1}, \\
k_{2}+a \omega_{20}^{2}=\left(k_{1}+k_{2}-c-b \omega_{20}^{2}\right) R_{2} .
\end{array}\right.
$$

In the state of static balance, to maintain the balance of the cantilever section, it is necessary to provide a static moment $\tau_{c 0}$ at the root, which is equal to the gravity moment. Therefore, there is the following equation:

$$
\tau_{c 0}=\frac{1}{2} m_{2} g l_{2} \cos \theta_{20}+m_{3} g\left(l_{2} \cos \theta_{20}+\frac{1}{2} l_{3} \cos \theta_{30}\right) .
$$

By combining equations (9), (15), and (16), the mass of equivalent rods $\left(m_{2}\right.$ and $\left.m_{3}\right)$ and the stiffness coefficients $\left(k_{1}\right.$ and $k_{2}$ ) of equivalent torsion springs can be obtained by the numerical solution.

\section{Equivalent Rigid Body Dynamic Model}

After obtaining the equivalent two-bar and two-spring mechanism of the cantilever section, the whole equivalent three-bar and two-spring mechanism can be constructed by considering the parameters of the root section of the arm.

3.1. The Complete Lagrange Dynamic Model. The translational kinetic energy of the three rods is

$$
T_{p i}=\frac{1}{2} m_{i}\left(\left(\frac{\mathrm{d} x_{c i}}{\mathrm{~d} t}\right)^{2}+\left(\frac{\mathrm{d} y_{c i}}{\mathrm{~d} t}\right)^{2}\right) \quad(i=1,2,3) .
$$

The rotational kinetic energy of the $i$ th rod around mass centers is

$$
T_{r i}=\frac{1}{6} m_{i} l_{c i}^{2} \dot{\theta}_{i}^{2} .
$$

The potential energy of three links is 


$$
\left\{\begin{array}{l}
V_{1}=\frac{1}{2} m_{1} g l_{1} \sin \theta_{1}, \\
V_{2}=m_{2} g\left(l_{1} \sin \theta_{1}+\frac{1}{2} l_{2} \sin \theta_{2}\right), \\
V_{3}=m_{3} g\left(l_{1} \sin \theta_{1}+l_{2} \sin \theta_{2}+\frac{1}{2} l_{3} \sin \theta_{3}\right) .
\end{array}\right.
$$

The elastic potential energy of two equivalent torsion springs is

$$
\left\{\begin{array}{l}
V_{k 1}=\frac{1}{2} k_{1}\left(\theta_{1}-\theta_{2}\right)^{2}, \\
V_{k 2}=\frac{1}{2} k_{2}\left(\theta_{2}-\theta_{3}\right)^{2} .
\end{array}\right.
$$

The total potential energy of the system is

$$
V=\sum_{1}^{3} V_{i}+\sum_{1}^{2} V_{k i}
$$

Substitute the terms in (17)-(21) into the Lagrange operator, and then use the Lagrange function to obtain $i$ th Lagrange (22) for the generalized coordinate $\theta_{i}$.

$$
\begin{aligned}
& \left\{\begin{array}{l}
\left(\frac{m_{1}}{3}+m_{2}+m_{3}\right) l_{1}^{2} \ddot{\theta}_{\delta 1}+m_{3} l_{1} l_{c 3}\left[\cos \left(\theta_{1}-\theta_{3}\right) \ddot{\theta}_{\delta 3}-\sin \left(\theta_{1}-\theta_{3}\right)\left(\dot{\theta}_{1}-\dot{\theta}_{3}\right) \dot{\theta}_{3}\right] \\
+\left(\frac{m_{2}}{2}+m_{3}\right) l_{1} l_{2}\left[\cos \left(\theta_{1}-\theta_{2}\right) \ddot{\theta}_{\delta 2}-\sin \left(\theta_{1}-\theta_{2}\right)\left(\dot{\theta}_{1}-\dot{\theta}_{2}\right) \dot{\theta}_{2}\right]+\left(\frac{m_{2}}{2}+m_{3}\right) l_{1} l_{2} \dot{\theta}_{1} \dot{\theta}_{2} \sin \left(\theta_{1}-\theta_{2}\right)
\end{array}\right. \\
& +\frac{m_{3}}{2} l_{1} l_{3} \dot{\theta}_{1} \dot{\theta}_{3} \sin \left(\theta_{1}-\theta_{3}\right)+\left(\frac{m_{1}}{2}+m_{2}+m_{3}\right) g l_{1} \cos \theta_{1}+k_{1}\left(\theta_{1}-\theta_{2}\right)=\tau, \\
& \left(\frac{m_{2}}{2}+m_{3}\right) l_{1} l_{2}\left[\cos \left(\theta_{1}-\theta_{2}\right) \ddot{\theta}_{\delta 1}-\sin \left(\theta_{1}-\theta_{2}\right)\left(\dot{\theta}_{1}-\dot{\theta}_{2}\right) \dot{\theta}_{1}\right]+\left(\frac{m_{2}}{3}+m_{3}\right) l_{2}^{2} \ddot{\theta}_{\delta 2}+\frac{m_{3}}{2} l_{2} l_{3} \dot{\theta}_{2} \dot{\theta}_{3} \sin \left(\theta_{2}-\theta_{3}\right) \\
& +\frac{m_{3}}{2} l_{2} l_{3}\left[\cos \left(\theta_{2}-\theta_{3}\right) \ddot{\theta}_{\delta 3}-\sin \left(\theta_{2}-\theta_{3}\right)\left(\dot{\theta}_{2}-\dot{\theta}_{3}\right) \dot{\theta}_{3}\right]-\left(\frac{m_{2}}{2}+m_{3}\right) l_{1} l_{2} \dot{\theta}_{1} \dot{\theta}_{2} \sin \left(\theta_{1}-\theta_{2}\right) \\
& +\left(\frac{m_{2}}{2}+m_{3}\right) l_{2} g \cos \theta_{2}-k_{1}\left(\theta_{1}-\theta_{2}\right)+k_{2}\left(\theta_{2}-\theta_{3}\right)=0 \\
& \frac{m_{3}}{2} l_{1} l_{3}\left[\cos \left(\theta_{1}-\theta_{3}\right) \ddot{\theta}_{\delta 1}-\sin \left(\theta_{1}-\theta_{3}\right)\left(\dot{\theta}_{1}-\dot{\theta}_{3}\right) \dot{\theta}_{1}\right]+\frac{m_{3}}{2} l_{2} l_{3}\left[\cos \left(\theta_{2}-\theta_{3}\right) \ddot{\theta}_{\delta 2}-\sin \left(\theta_{2}-\theta_{3}\right)\left(\dot{\theta}_{2}-\dot{\theta}_{3}\right) \dot{\theta}_{2}\right] \\
& +\frac{m_{3}}{3} l_{3}^{2} \ddot{\theta}_{\delta 3}-\frac{m_{3}}{2} l_{1} l_{3} \dot{\theta}_{1} \dot{\theta}_{3} \sin \left(\theta_{1}-\theta_{3}\right)-\frac{m_{3}}{2} l_{2} l_{3} \dot{\theta}_{2} \dot{\theta}_{3} \sin \left(\theta_{2}-\theta_{3}\right)+\frac{m_{3}}{2} g l_{3} \cos \theta_{3}-k_{2}\left(\theta_{2}-\theta_{3}\right)=0
\end{aligned}
$$

3.2. Linear Approximation. It is assumed that each angle $\theta_{i}$ vibrates in a small range near its static position $\theta_{i 0}$. And when the springs are in the state of static balance, the following equation exists:

$$
\left\{\begin{array}{l}
k_{1}\left(\theta_{10}-\theta_{20}\right)=m_{2} g l_{c 2} \cos \theta_{20}+m_{3} g\left(l_{2} \cos \theta_{20}+l_{c 3} \cos \theta_{30}\right), \\
k_{2}\left(\theta_{20}-\theta_{30}\right)=m_{3} g l_{c 3} \cos \theta_{30} .
\end{array}\right.
$$


The static balance torque of the first link required to maintain the balance state is

$$
\tau_{0}=m_{1} g l_{c 1} \cos \theta_{10}+m_{2} g\left(l_{1} \cos \theta_{10}+l_{c 2} \cos \theta_{20}\right)+m_{3} g\left(l_{1} \cos \theta_{10}+l_{2} \cos \theta_{20}++l_{c 3} \cos \theta_{30}\right) \text {. }
$$

The torque increment is $\tau_{\delta}=\tau-\tau_{0}$. Ignoring the higherorder infinitesimal and then substituting (23) and (24) into (22), it will be expressed as

$$
\left\{\begin{array}{l}
a_{1} \ddot{\theta}_{\delta 3}+a_{2} \ddot{\theta}_{\delta 2}+a_{3} \ddot{\theta}_{\delta 1}+a_{4} \theta_{\delta 2}+a_{5} \theta_{\delta 1}=\tau_{\delta}, \\
a_{6} \ddot{\theta}_{\delta 3}+a_{7} \ddot{\theta}_{\delta 2}+a_{2} \ddot{\theta}_{\delta 1}+a_{8} \theta_{\delta 3}+a_{9} \theta_{\delta 2}+a_{4} \theta_{\delta 1}=0, \\
a_{10} \ddot{\theta}_{\delta 3}+a_{6} \ddot{\theta}_{\delta 2}+a_{1} \ddot{\theta}_{\delta 1}+a_{11} \theta_{\delta 3}+a_{8} \theta_{\delta 2}=0,
\end{array}\right.
$$

where

$$
\left\{\begin{array}{l}
a_{1}=\frac{1}{2} m_{3} l_{1} l_{3}, a_{2}=\left(\frac{1}{2} m_{2}+m_{3}\right) l_{1} l_{2}, \\
a_{3}=\left(\frac{1}{3} m_{1}+m_{2}+m_{3}\right) l_{1}^{2}, \\
a_{4}=-k_{1}, \\
a_{5}=k_{1}-\left(\frac{1}{2} m_{1}+m_{2}+m_{3}\right) g l_{1} \sin \theta_{10} \\
a_{6}=\frac{1}{2} m_{3} l_{2} l_{3}, \\
a_{7}=\left(\frac{1}{3} m_{2}+m_{3}\right) l_{2}^{2}, \\
a_{10}=\frac{1}{3} m_{3} l_{3}^{2}, a_{11}=k_{2}-\frac{1}{2} m_{3} g l_{3} \sin \theta_{30} \\
a_{9}=-k_{2}, \\
a_{1}+k_{2}-\left(\frac{1}{2} m_{2}+m_{3}\right) g l_{2} \sin \theta_{20},
\end{array}\right.
$$

\section{Determination of the DF Output}

For the single-input multiple-output (SIMO) model, the DF method is used to find an output, and the general controller is used to achieve the purpose of LFA vibration suppression. Under this idea, the dynamic relationship between the output variables in the underactuated system can make the system into a SISO form, which can be easily controlled.
4.1. Combination of 3 Tilt Angles. Equation (25) can be written as

$$
\mathbf{M} \ddot{\mathbf{q}}=\left[\begin{array}{c}
\tau_{\delta} \\
0 \\
0
\end{array}\right]-\mathbf{N q},
$$

while

$$
\begin{aligned}
\mathbf{q} & =\left[\begin{array}{l}
\theta_{\delta 3} \\
\theta_{\delta 2} \\
\theta_{\delta 1}
\end{array}\right], \\
\ddot{\mathbf{q}} & =\left[\begin{array}{l}
\ddot{\theta}_{\delta 3} \\
\ddot{\theta}_{\delta 2} \\
\ddot{\theta}_{\delta 1}
\end{array}\right], \\
\mathbf{M} & =\left[\begin{array}{lll}
a_{1} & a_{2} & a_{3} \\
a_{6} & a_{7} & a_{2} \\
a_{10} & a_{6} & a_{1}
\end{array}\right], \\
\mathbf{N} & =\left[\begin{array}{lll}
a_{4} & a_{5} \\
a_{8} & a_{9} & a_{4} \\
a_{11} & a_{8} & 0
\end{array}\right] .
\end{aligned}
$$

Premultiplying both sides of (27) by the inverse matrix $\mathbf{M}^{-1}$, it will be

$$
\ddot{\mathbf{q}}=\frac{\widetilde{Q}}{\mathrm{Q}}\left(\left[\begin{array}{c}
\tau_{\delta} \\
0 \\
0
\end{array}\right]-\mathbf{N q}\right),
$$

where

$$
\begin{aligned}
& \widetilde{Q}=\left[\begin{array}{ccc}
a_{1} a_{7}-a_{2} a_{6} & a_{3} a_{6}-a_{1} a_{2} & a_{2}^{2}-a_{3} a_{7} \\
a_{2} a_{10}-a_{1} a_{6} & a_{1}^{2}-a_{3} a_{10} & a_{3} a_{6}-a_{1} a_{2} \\
a_{6}^{2}-a_{7} a_{10} & a_{2} a_{10}-a_{1} a_{6} & a_{1} a_{7}-a_{2} a_{6}
\end{array}\right], \\
& Q=a_{1}^{2} a_{7}-2 a_{1} a_{2} a_{6}+a_{2}^{2} a_{10}+a_{6}^{2} a_{3}-a_{3} a_{7} a_{10} .
\end{aligned}
$$

In order to control the arm to be stable in a certain attitude, the three elevation angles will be balanced at a special situation under an angle command, so the linear combination of three tilt angle increments is used to form a differential flatness output, namely,

$$
F=\mu_{2} \theta_{\delta 3}+\mu_{1} \theta_{\delta 2}+\theta_{\delta 1} .
$$

Premultiplying by $\left(\begin{array}{lll}\mu_{2} & \mu_{1} & 1\end{array}\right)$ at both sides of (29), the result is 


$$
\ddot{F}=\frac{\widetilde{Q}}{Q}\left(\begin{array}{lll}
\mu_{2} & \mu_{1} & 1
\end{array}\right)\left(\left[\begin{array}{c}
\tau_{\delta} \\
0 \\
0
\end{array}\right]-\mathbf{N q}\right) .
$$

In addition, the second derivative for both sides of (31) with respect to time is

$$
\ddot{F}=\mu_{2} \ddot{\theta}_{\delta 3}+\mu_{1} \ddot{\theta}_{\delta 2}+\ddot{\theta}_{\delta 1} \text {. }
$$

4.2. Parameters of the DF Output. The core of the DF method is that the selected flatness output and its continuous multiorder derivatives can represent the $m$ state variables and $n$ input variables algebraically as a basis vector. It means that the flatness output and its derivatives are linearly independent, which can represent all the original state variables and input variables linearly to satisfy this condition [19]. Therefore, the flatness processing is to find an output $y$ and its $(m+n-1)$ derivatives so that the $(m+n)$-dimensional basis vector elements can represent all the state variables and input variables linearly. Here are three angular outputs and three derivatives of the angular outputs and a force input in formula (25), a total of seven variables must be expressed linearly by the vector basis of the flatness output $F$. Therefore, the differential flat output and at least the six-order differentiations are needed to decouple the original system.

On the contrary, $\tau_{\delta}$ is the only input; this means, under the only input, the other six variables must respond concurrently. In short, $\theta_{\delta i}$ must be expressed by the fourth-order or much lower differential terms of $F$; otherwise, the secondorder derivative term of angles must be expressed as a linear combination of more than six-order differential terms of $F$. When equation (33) contains only the second derivative of $F$ and the increment of each angle, the system input cannot be decoupled in this case. So, the solution to equation (32) is to use the following constraints:

$$
\mu_{2}=\frac{a_{6}^{2}-a_{7} a_{10}+\left(a_{2} a_{10}-a_{1} a_{6}\right) \mu_{1}}{a_{2} a_{6}-a_{1} a_{7}} .
$$

Under such a condition, (32) is transformed into (30).

$\ddot{F}=-\frac{\left(a_{9} Q_{1}+a_{15} Q_{2}\right) \theta_{\delta 3}+\left(a_{10} Q_{1}+a_{9} Q_{2}\right) \theta_{\delta 2}+a_{4} Q_{1} \theta_{\delta 1}}{Q}$, where

$$
\left\{\begin{array}{l}
Q_{1}=\left(a_{3} a_{6}-a_{1} a_{2}\right) \mu_{2}+\left(a_{1}^{2}-a_{3} a_{10}\right) \mu_{1}+a_{2} a_{10}-a_{1} a_{6}, \\
Q_{2}=\left(a_{2}^{2}-a_{3} a_{7}\right) \mu_{2}+\left(a_{3} a_{6}-a_{1} a_{2}\right) \mu_{1}+a_{1} a_{7}-a_{2} a_{6} .
\end{array}\right.
$$

Let $Q_{1}=p Q, p \in \mathbb{R}$; we can get

$$
Q_{2}=\frac{\left(1-p a_{2}\right) Q}{a_{1}} \text {. }
$$

In this way, two undetermined coefficients can be represented only by $p$ in the following equation:

$$
\left\{\begin{array}{l}
\mu_{1}=\frac{a_{6}+\left(a_{1} a_{7}-a_{2} a_{6}\right) p}{a_{1}}, \\
\mu_{2}=\frac{a_{10}+\left(a_{1} a_{6}-a_{2} a_{10}\right) p}{a_{1}} .
\end{array}\right.
$$

Substituting (38) into (32), we can get

$$
\ddot{F}=n_{3} \theta_{\delta 3}+n_{2} \theta_{\delta 2}+n_{1} \theta_{\delta 1},
$$

where

$$
\left\{\begin{array}{l}
n_{1}=-p a_{4}, \\
n_{2}=\frac{\left(a_{2} a_{8}-a_{1} a_{9}\right) p-a_{8}}{a_{1}}, \\
n_{3}=\frac{\left(a_{2} a_{11}-a_{1} a_{8}\right) p-a_{11}}{a_{1}} .
\end{array}\right.
$$

Taking the second derivative of both sides of equation (39) gives the following result:

$$
F^{(4)}=n_{3} \ddot{\theta}_{\delta 3}+n_{2} \ddot{\theta}_{\delta 2}+n_{1} \ddot{\theta}_{\delta 1} .
$$

The first and third forms of (27) are combined with (39) and are arranged into the following form:

$$
\left\{\begin{array}{l}
a_{1} \ddot{\theta}_{\delta 3}+a_{2} \ddot{\theta}_{\delta 2}+a_{3} \ddot{\theta}_{\delta 1}=\tau_{\delta}-a_{4} \theta_{\delta 2}-a_{5} \theta_{\delta 1}, \\
a_{10} \ddot{\theta}_{\delta 3}+a_{6} \ddot{\theta}_{\delta 2}+a_{1} \ddot{\theta}_{\delta 1}=-a_{11} \theta_{\delta 3}-a_{8} \theta_{\delta 2}, \\
{\left[p\left(a_{1} a_{6}-a_{2} a_{10}\right)+a_{10}\right] \ddot{\theta}_{\delta 3}+\left[p\left(a_{1} a_{7}-a_{2} a_{6}\right)+a_{6}\right] \ddot{\theta}_{\delta 2}+a_{1} \ddot{\theta}_{\delta 1}=a_{1} \ddot{F}}
\end{array} .\right.
$$

By solving equation (42), $\ddot{\theta}_{\delta i}$ can be expressed by $\theta_{\delta i}, \tau_{\delta}$ in the formula which contains the unknown variable $p$. Substitute $\ddot{\theta}_{\delta i}$ into equation (41) to obtain the expression of $F^{(4)}$. According to the need of solving, only the coefficient of $\tau_{\delta}$ in this expression is listed here. 


$$
\frac{a_{1} a_{6}\left(a_{8}+a_{1} a_{9} p\right)-a_{11}\left(a_{2} a_{6}-a_{1} a_{7}\right)\left(a_{2} p-1\right)+a_{10}\left[a_{1} a_{4} a_{7} p+a_{2}^{2} a_{8} p-a_{2}\left(a_{8}+a_{1} a_{9} p\right)\right]}{a_{3} a_{6}^{2}-2 a_{1} a_{2} a_{6}+a_{7} a_{1}^{2}+a_{10}\left(a_{2}^{2}-a_{3} a_{7}\right)} .
$$

Taking the same way as (34), let coefficient (43) be zero to eliminate the possible decoupling relationship between the fourth derivative of output $F$ and the input torque. Then, we can get

$$
p=\frac{a_{1} a_{7} a_{11}+a_{2} a_{8} a_{10}-a_{2} a_{6} a_{11}-a_{1} a_{6} a_{8}}{a_{1} a_{2} a_{7} a_{11}-a_{1} a_{2} a_{9} a_{10}+a_{1} a_{4} a_{7} a_{10}-a_{1} a_{4} a_{6}^{2}+a_{2}^{2}\left(a_{8} a_{10}-a_{6} a_{11}\right)+a_{1}^{2}\left(a_{6} a_{9}-a_{7} a_{8}\right)} .
$$

The explicit expression of $F$ can be obtained by substituting the value of $p$ into (33), and then substitute the where value of $p$ into (41) to get the following equation:

$$
\left\{\begin{array}{l}
n_{4}=\frac{a_{4} a_{6} a_{11}-a_{4} a_{8} a_{10}+a_{1} a_{8}^{2}-a_{1} a_{9} a_{11}}{a_{1} a_{7} a_{11}+a_{2} a_{8} a_{10}-a_{2} a_{6} a_{11}-a_{1} a_{6} a_{8}}, \\
n_{5}=\frac{a_{8}}{a_{1}}\left[b_{4}+\frac{a_{4}\left(a_{1} a_{7} a_{11}+a_{2} a_{8} a_{10}-a_{2} a_{6} a_{11}-a_{1} a_{6} a_{8}\right)}{a_{1} a_{4}\left(a_{7} a_{10}-a_{6}^{2}\right)+a_{2}^{2}\left(a_{8} a_{10}-a_{6} a_{11}\right)+a_{1}^{2}\left(a_{6} a_{9}-a_{7} a_{8}\right)+a_{1} a_{2}\left(a_{7} a_{11}-a_{9} a_{10}\right)}\right], \\
b_{6}=\frac{a_{11} b_{5}}{a_{8}} .
\end{array}\right.
$$

Taking the second derivative on both sides of (46),

$$
F^{(6)}=n_{6} \ddot{\theta}_{\delta 3}+n_{5} \ddot{\theta}_{\delta 2}+n_{4} F^{(4)} \text {. }
$$

By solving equations (31), (33), (39), (41), (45), and (47) simultaneously, the results can be obtained.

$$
\left\{\begin{array}{l}
\ddot{\theta}_{\delta 3}=\frac{-n_{1} n_{5} \ddot{F}+\left(n_{2} n_{4}+n_{5}-n_{1} n_{4} \mu_{1}\right) F^{(4)}+\left(n_{1} \mu_{1}-n_{2}\right) F^{(6)}}{n_{3} n_{5}-n_{2} n_{6}+n_{1} n_{6} \mu_{1}-n_{1} n_{5} \mu_{2}}, \\
\ddot{\theta}_{\delta 2}=\frac{n_{1} n_{6} \ddot{F}+\left(n_{1} n_{4} \mu_{2}-n_{3} n_{4}-n_{6}\right) F^{(4)}+\left(n_{3}-n_{1} \mu_{2}\right) F^{(6)}}{n_{3} n_{5}-n_{2} n_{6}+n_{1} n_{6} \mu_{1}-n_{1} n_{5} \mu_{2}} . \\
\ddot{\theta}_{\delta 1}=\frac{\left(n_{3} n_{5}-n_{2} n_{6}\right) \ddot{F}+\left[\left(n_{3} n_{4}+n_{6}\right) \mu_{1}-\left(n_{2} n_{4}+n_{5}\right) \mu_{2}\right] F^{(4)}+\left(n_{2} \mu_{2}-n_{3} \mu_{1}\right) F^{(6)}}{n_{3} n_{5}-n_{2} n_{6}+n_{1} n_{6} \mu_{1}-n_{1} n_{5} \mu_{2}} \\
\theta_{\delta 3}=\frac{-n_{1} n_{5} F+\left(n_{2} n_{4}+n_{5}-n_{1} n_{4} \mu_{1}\right) F^{(2)}+\left(n_{1} \mu_{1}-n_{2}\right) F^{(4)}}{n_{3} n_{5}-n_{2} n_{6}+n_{1} n_{6} \mu_{1}-n_{1} n_{5} \mu_{2}} \\
\theta_{\delta 2}=\frac{n_{1} n_{6} F+\left(n_{1} n_{4} \mu_{2}-n_{3} n_{4}-n_{6}\right) F^{(2)}+\left(n_{3}-n_{1} \mu_{2}\right) F^{(4)}}{n_{3} n_{5}-n_{2} n_{6}+n_{1} n_{6} \mu_{1}-n_{1} n_{5} \mu_{2}}, \\
\theta_{\delta 1}=\frac{\left(n_{3} n_{5}-n_{2} n_{6}\right) F+\left[\left(n_{3} n_{4}+n_{6}\right) \mu_{1}-\left(n_{2} n_{4}+n_{5}\right) \mu_{2}\right] F^{(2)}+\left(n_{2} \mu_{2}-n_{3} \mu_{1}\right) F^{(4)}}{n_{3} n_{5}-n_{2} n_{6}+n_{1} n_{6} \mu_{1}-n_{1} n_{5} \mu_{2}}
\end{array} .\right.
$$


Substituting the variables in (48) into the first formula in where (25), we can get

$$
F^{(6)}+d_{1} F^{(4)}+d_{2} \ddot{F}+d_{3} F=d_{4} \tau_{\delta},
$$

$$
\left\{\begin{array}{l}
d_{1}=\frac{a_{2}\left(b_{1} b_{4}+b_{6}\right)-a_{1}\left(b_{2} b_{4}+b_{5}\right)-a_{4} b_{3}+\left[a_{5} b_{3}+a_{1} b_{1} b_{4}-a_{3}\left(b_{3} b_{4}+b_{6}\right)\right] \mu_{1}+\left[a_{4} b_{1}-a_{2} b_{1} b_{4}-a_{5} b_{2}+a_{3}\left(b_{2} b_{4}+b_{5}\right)\right] \mu}{a_{1} b_{2}-a_{2} b_{3}+\left(a_{3} b_{3}-a_{1} b_{1}\right) \mu_{1}+\left(a_{2} b_{1}-a_{3} b_{2}\right) \mu_{2}} \\
d_{2}=\frac{a_{4}\left(b_{3} b_{4}+b_{6}\right)+a_{3}\left(b_{2} b_{6}-b_{3} b_{5}\right)+b_{1}\left(a_{1} b_{5}-a_{2} b_{6}\right)-a_{5}\left(b_{6}+b_{3} b_{4}\right) \mu_{1}+\left(a_{5} b_{5}+a_{5} b_{2} b_{4}-a_{4} b_{1} b_{4}\right) \mu_{2}}{a_{1} b_{2}-a_{2} b_{3}+\left(a_{3} b_{3}-a_{1} b_{1}\right) \mu_{1}+\left(a_{2} b_{1}-a_{3} b_{2}\right) \mu_{2}} \\
d_{3}=\frac{a_{5} b_{2} b_{6}-a_{5} b_{3} b_{5}-a_{4} b_{1} b_{6}}{a_{1} b_{2}-a_{2} b_{3}+\left(a_{3} b_{3}-a_{1} b_{1}\right) \mu_{1}+\left(a_{2} b_{1}-a_{3} b_{2}\right) \mu_{2}} \\
d_{4}=\frac{b_{2} b_{6}-b_{3} b_{5}-b_{1} b_{6} \mu_{1}+b_{1} b_{5} \mu_{2}}{a_{1} b_{2}-a_{2} b_{3}+\left(a_{3} b_{3}-a_{1} b_{1}\right) \mu_{1}+\left(a_{2} b_{1}-a_{3} b_{2}\right) \mu_{2}} .
\end{array}\right.
$$

So far, all the angles and their second derivatives in the dynamic model of the three-bar and two-spring mechanism can be expressed linearly by $F$ and its first six derivatives; that is, when $F$ is used as the controlled variable, the system is flat, that is, the system is controllable.

\section{The ADRC System Based on the DF Output}

The research of ADRC has experienced 30 years of exploration. A large number of studies have proved that dynamic systems, linear or nonlinear, under some conditions can be transformed into the canonical form of cascade integrators via feedback $[20,21]$. This makes the control system have excellent ability to resist internal and external disturbances, even if the information of the model is very scarce.

The vibration-suppressing control system for the LFA based on ADRC, as shown in Figure 2, is constructed after the output of the controlled object is synthesized into the DF output.

We are more focused on the fast stabilization method of the low-frequency large-amplitude oscillation. In order to avoid the more complex response caused by substituting the high-order terms into the controller and make full use of ESO's strong points [16], the high-order term together with the error caused by the linearization of the model and other neglected unmodeled uncertainties $(\psi)$ and unknown external disturbance $(D)$ are regarded as the integrated disturbance; that is, $f=D+\zeta+d_{1} F^{(4)}+F^{(6)}$.

Then, formula (49) can be written as

$$
\ddot{F}=b_{0} \tau_{\delta}-b_{1} F+f_{\left(D, \zeta, F^{(4)}, F^{(6)}\right)},
$$

where

$$
\left\{\begin{array}{l}
b_{1}=\frac{a_{5}\left(n_{2} n_{6}-n_{3} n_{5}\right)-a_{4} n_{1} n_{6}}{a_{4} n_{3} n_{4}+a_{1} n_{1} n_{5}-a_{3} n_{3} n_{5}+a_{4} n_{6}-a_{2} n_{1} n_{6}+a_{3} n_{2} n_{6}-\left(a_{5} n_{6}+a_{5} n_{3} n_{4}\right) \mu_{1}+\left(a_{5} b_{5}+a_{5} n_{2} n_{4}-a_{4} n_{1} n_{4}\right) \mu_{2}}, \\
b_{0}=\frac{n_{2} n_{6}-n_{3} n_{5} a_{5}-n_{1} n_{6} \mu_{1}+n_{1} n_{5} \mu_{2}}{a_{4} n_{3} n_{4}+a_{1} n_{1} n_{5}-a_{3} n_{3} n_{5}+a_{4} n_{6}-a_{2} n_{1} n_{6}+a_{3} n_{2} n_{6}-\left(a_{5} n_{6}+a_{5} n_{3} n_{4}\right) \mu_{1}+\left(a_{5} b_{5}+a_{5} n_{2} n_{4}-a_{4} n_{1} n_{4}\right) \mu_{2}} .
\end{array}\right.
$$

5.1. The Extended State Observer (ESO). We refer to the basis of linear ADRC theory $[22,23]$; consider $f$ as an extended state, and it is differentiable; that is, $\dot{f}=h, h \in \mathbb{R}$.

Then, the extended system can be expressed as

$$
\left\{\begin{array}{l}
\dot{\mathbf{x}}=\mathbf{A} \mathbf{x}+\mathbf{B} \tau_{\delta}+\mathbf{E} h \\
y=\mathbf{C} x
\end{array}\right.
$$

where 


$$
\begin{aligned}
& \mathbf{x}=\left[\begin{array}{c}
F \\
\dot{F} \\
f
\end{array}\right], \\
& \mathbf{A}=\left[\begin{array}{ccc}
0 & 1 & 0 \\
-b_{1} & 0 & 1 \\
0 & 0 & 0
\end{array}\right], \\
& \mathbf{B}=\left[\begin{array}{c}
0 \\
b_{0} \\
0
\end{array}\right], \\
& \mathbf{C}=\left[\begin{array}{lll}
1 & 0 & 0
\end{array}\right], \\
& \mathbf{E}=\left[\begin{array}{lll}
0 & 0 & 1
\end{array}\right]^{T} .
\end{aligned}
$$

The Luenberger observer shown in equation (55) is used to observe the state of system (53) and the extended state variable $f$.

$$
\left\{\begin{array}{l}
\dot{\mathbf{z}}=\mathbf{A} \widehat{\mathbf{z}}+\mathbf{B} \tau_{\delta}+\mathbf{L}(y-\widehat{y}), \\
\hat{y}=\mathbf{C} \widehat{\mathbf{z}} .
\end{array}\right.
$$

The linear ADRC structure is used to place the poles at $\left(-\omega_{o}\right)$, while $\omega_{o}$ is named as the bandwidth of the observer. It means $|\lambda \mathbf{I}-(\mathbf{A}-\mathbf{L C})|=\left(\lambda+\omega_{o}\right)^{3}$.

Then, the observer gain is

$$
\left\{\begin{array}{l}
L_{1}=3 \omega_{o} \\
L_{2}=3 \omega_{o}^{2}-b_{1}, \\
L_{3}=\omega_{o}^{3} .
\end{array}\right.
$$

5.2. The Controller. Based on observer (55) configured by (56), the system (A-BK) is asymptotically stable, which means that the generalized disturbance estimated by the observer can be used for compensation in the controller so that the generalized disturbance itself can be rejected. By using the reasonable control law for the feedback control, the system will be simplified as a canonical form of cascade integrators.

$$
F_{(t)} \approx \iint \tau_{0(t)} \mathrm{d} t
$$

Therefore, the control law is designed as

$$
\tau_{\delta(t)}=\widetilde{\mathbf{K}} \mathbf{z} .
$$

Use (59) to set the eigenvalue of the feedback controller $(\mathrm{A}-\mathrm{BK})$ at $\left(-\omega_{c}\right)$.

$$
\widetilde{\mathbf{K}}=\frac{1}{b_{0}}\left[\left(\begin{array}{lll}
\left.\omega_{c}^{2}-b_{1}\right) & 2 \omega_{c} & 1
\end{array}\right] .\right.
$$

By substituting right model information into the controller, the tuning range will be smaller; more

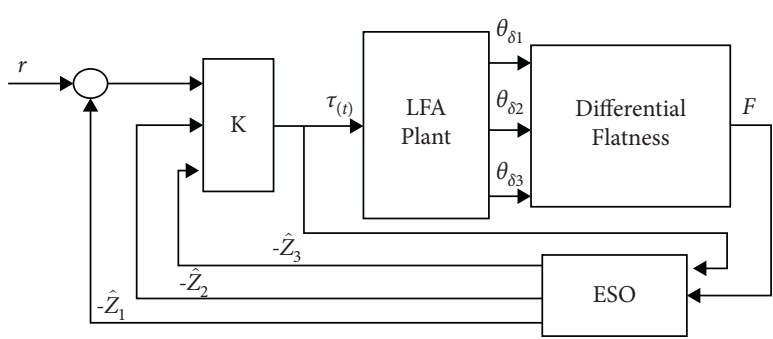

FIgURE 2: DF-based ADRC implementation framework.

importantly, the burden of the observer can be effectively reduced $[24,25]$. The tuning of system parameters becomes simple; only $\omega_{c}$ and $\omega_{\text {o }}$ need to be adjusted. To a certain extent, the wider the stability threshold of the observer is, the more stable the system is. This method, also known as the grey box method, is a way to utilize model information as much as possible in the model-free control process, which is also a beneficial development of ADRC thinking.

\section{The Prototype Experiment Based on the DF Output}

Equipment required for mechanized pollination of high branches in agricultural production is shown in Figure 3. The working scenarios of the LFA include high crown pruning, flower thinning, and picking. The overall arm length is $4.9 \mathrm{~m}$, the length of the cantilever section above the hinge point of the electric cylinder is $4.2 \mathrm{~m}$, the hollow square aluminum tube used is $30 \mathrm{~mm}$ in section width, $20 \mathrm{~mm}$ in section height, and $2 \mathrm{~mm}$ in wall thickness, and the section moment of inertia is $I=1.1125 \times 10^{-8} \mathrm{~m}^{4}$.

\subsection{The Equivalent of the Cantilever Part}

6.1.1. Determination of the Rod Length and Sensor Installation Position. The cantilever section of the research object is a structure with uniform material and constant cross section, and the first two natural frequencies are obtained by using the partial differential equation method [26].

$$
\omega_{i 0}=\frac{\left(\beta_{i} l\right)^{2}}{l^{2}} \sqrt{\frac{E I}{\bar{m}}}
$$

where material elastic modulus is $E=27 \mathrm{GPa}$, length density is $\bar{m}=0.502 \mathrm{~kg} / \mathrm{m}$, and the constant terms $\beta_{1} l=1.875$ and $\beta_{2} l=4.694$. It can be concluded that the natural angular frequencies of the first two modes are

$$
\left\{\begin{array}{l}
\omega_{10}=4.88 \mathrm{rad} / \mathrm{s} \\
\omega_{20}=30.6 \mathrm{rad} / \mathrm{s}
\end{array}\right.
$$

The function of the first two modes is 


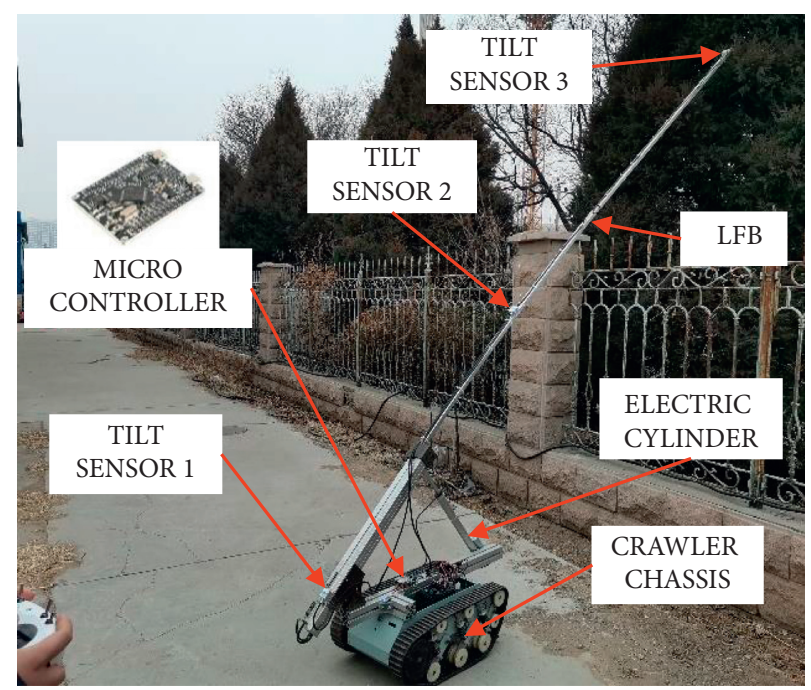

Figure 3: Experimental prototype of the LFA.

$$
W_{i(x)}=\cosh \beta_{i} x-\cos \beta_{i} x-\frac{\cosh \beta_{i} l+\cos \beta_{i} l}{\sinh \beta_{i} l+\sin \beta_{i} l}\left(\sinh \beta_{i} x+\sin \beta_{i} x\right), \quad x \in[0,1], i=1,2 .
$$

The mode function of the second mode and its first and second derivative curves are shown in Figure 4. The first derivative of the second mode equation is zero at point $A_{1}$ with an extension of $1.98 \mathrm{~m}$. Then, according to the point where the second derivative of the second mode function is zero, the point with the most significant change of the rotation angle in the vibration process is determined as the installation position of tilt sensor 2 . The second derivative of the second-order mode curve equation of the beam is zero at the $A_{2}$ point which is $0.91 \mathrm{~m}$ away from zero; it means $l_{k 1}=0.91 \mathrm{~m}$, and tilt sensor 3 is installed at the end of the beam.

6.1.2. Determination of Equivalent Model Parameters. The static deformation of the cantilever part of the LFA under the effect of self-weight can be obtained by finite element analysis or direct analysis or experimental measurement for the beam with constant cross section. In this paper, a statically balanced attitude is selected randomly. The optional elevation angles corresponding to each sensor position are

$$
\begin{aligned}
& \theta_{10}=0.88094 \mathrm{rad}, \\
& \theta_{20}=0.80419 \mathrm{rad}, \\
& \theta_{30}=0.77453 \mathrm{rad} .
\end{aligned}
$$

According to the Euler beam theory, the derivative of the deflection curve of the beam can express the angle of the section, so the derivative of the mode function can express the natural mode of vibration in the form of angle. The first derivative of the first-order mode function of the cantilever section of the LFA is calculated by using (62), and the ratio of angular mode function at $A_{2}$ and $A_{1}$ is $R_{1}=1.47$. Similarly, taking the first derivative of the second-order mode function, the angle ratio between $A_{2}$ and $A_{1}$ is $R_{2}=-2.05$.
Equations (9), (16), and (18) contain two equivalent masses and two equivalent torsion spring stiffness. In addition, the part between the two hinged joints at the bottom of the long arm is relatively strong, so it is regarded as a rigid segment, and its mass and length are directly measured. In this way, eight necessary parameters of the equivalent model for the rods and springs of the LFA are determined quickly and effectively, as shown in Table 1.

\subsection{The Experiment and Result}

6.2.1. Determination of the Parameter for the DF Output. By substituting the constants in Table 1 into (44), the value of $p$ is 0.3562 , and the coefficients of DF can be obtained: $\mu_{1}=2.544$ and $\mu_{2}=0.879$. Due to the influence of elevation, operating load, and other conditions, the static balance condition under different attitudes is not easy to specify continuously and accurately. It is unrealistic and unreliable to set the target angle for the sensor position corresponding to each equivalent bar. Under the assumption that the vibration process is small angle deformation, an overall target elevation angle $\theta_{0}$ is used as the unified target for the three rods. In this way, we can get the differential flat output as $F=\theta_{1}-\theta_{0}+2.544\left(\theta_{2}-\theta_{0}\right)+0.879\left(\theta_{3}-\theta_{0}\right)$. The purpose is to make $F$ close to zero as soon as possible by controlling the torque $\tau_{\delta}$.

6.2.2. Uncontrolled Free Vibration. An impact is applied in the middle of the LFA, and a swing of about $9^{\circ}$ amplitude is produced at the endpoint while keeping the drive motor locked during the experiment. Figure 5 depicts the process of free vibration and gradual attenuation. Since there is no complete constraint between the track chassis and the ground, it can be seen from the data of tilt sensor 1 that the 

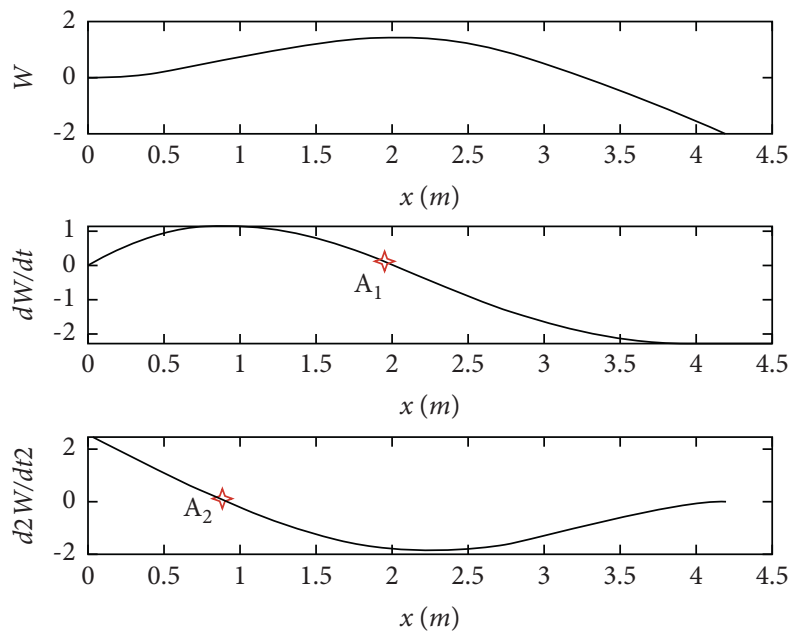

Figure 4: The second mode curve and its derivative curve.

TABle 1: Parameters of the equivalent mechanism.

\begin{tabular}{lcc}
\hline Symbol & Value & \\
\hline$m_{1}$ & 5.85 & $\mathrm{Unit}$ \\
$m_{2}$ & 1.4235 & $\mathrm{~kg}$ \\
$m_{3}$ & 0.663 & $\mathrm{~kg}$ \\
$l_{1}$ & 0.7 & $\mathrm{~kg}$ \\
$l_{2}$ & 1.98 & $\mathrm{~m}$ \\
$l_{3}$ & 2.22 & $\mathrm{~m}$ \\
$k_{1}$ & 779.8012 & $\mathrm{~m}$ \\
$k_{2}$ & 946.1959 & $\mathrm{~N} \cdot \mathrm{m} / \mathrm{rad}$ \\
\hline
\end{tabular}

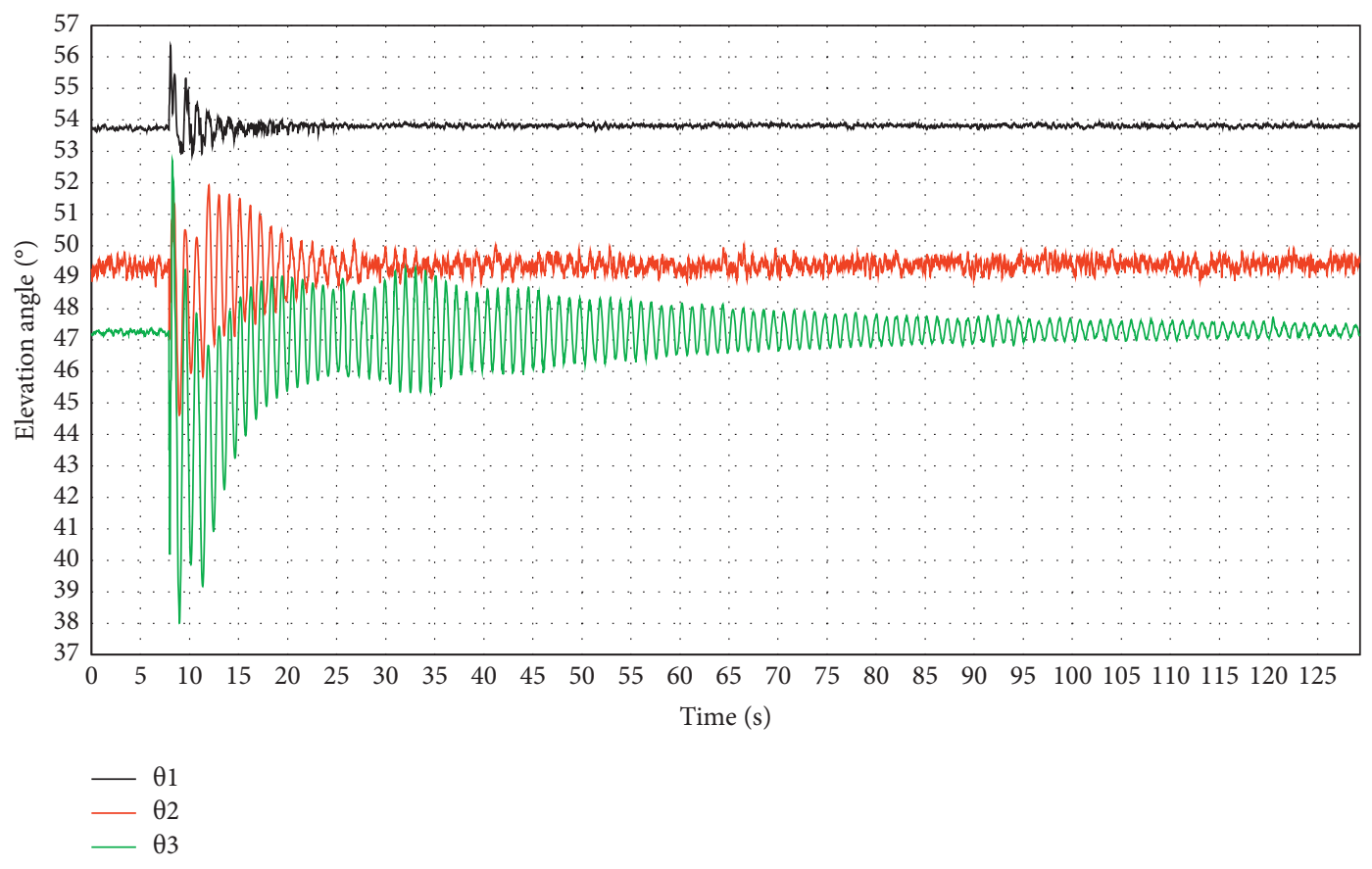

FIgURE 5: Free vibration curve with no control. 


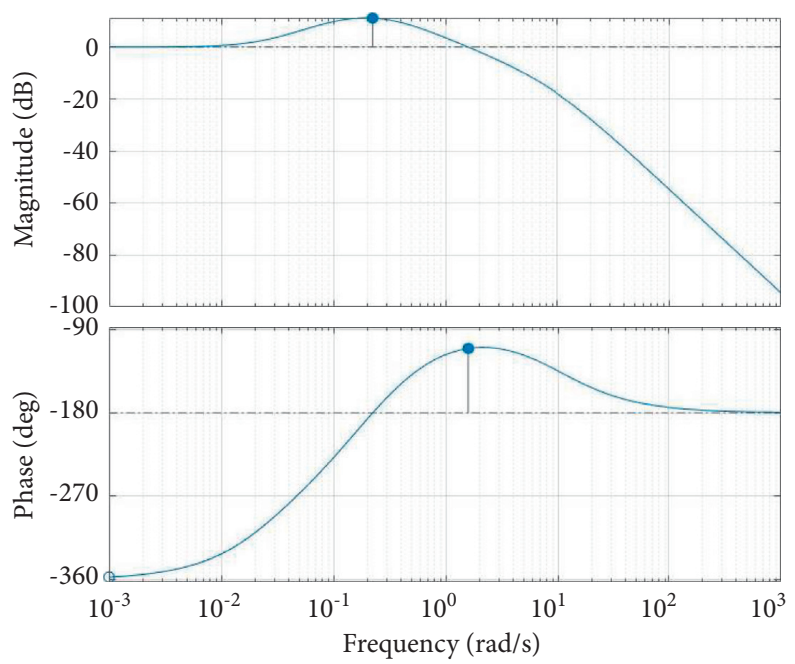

Figure 6: Bode diagrams for closed-loop systems with the PID controller.

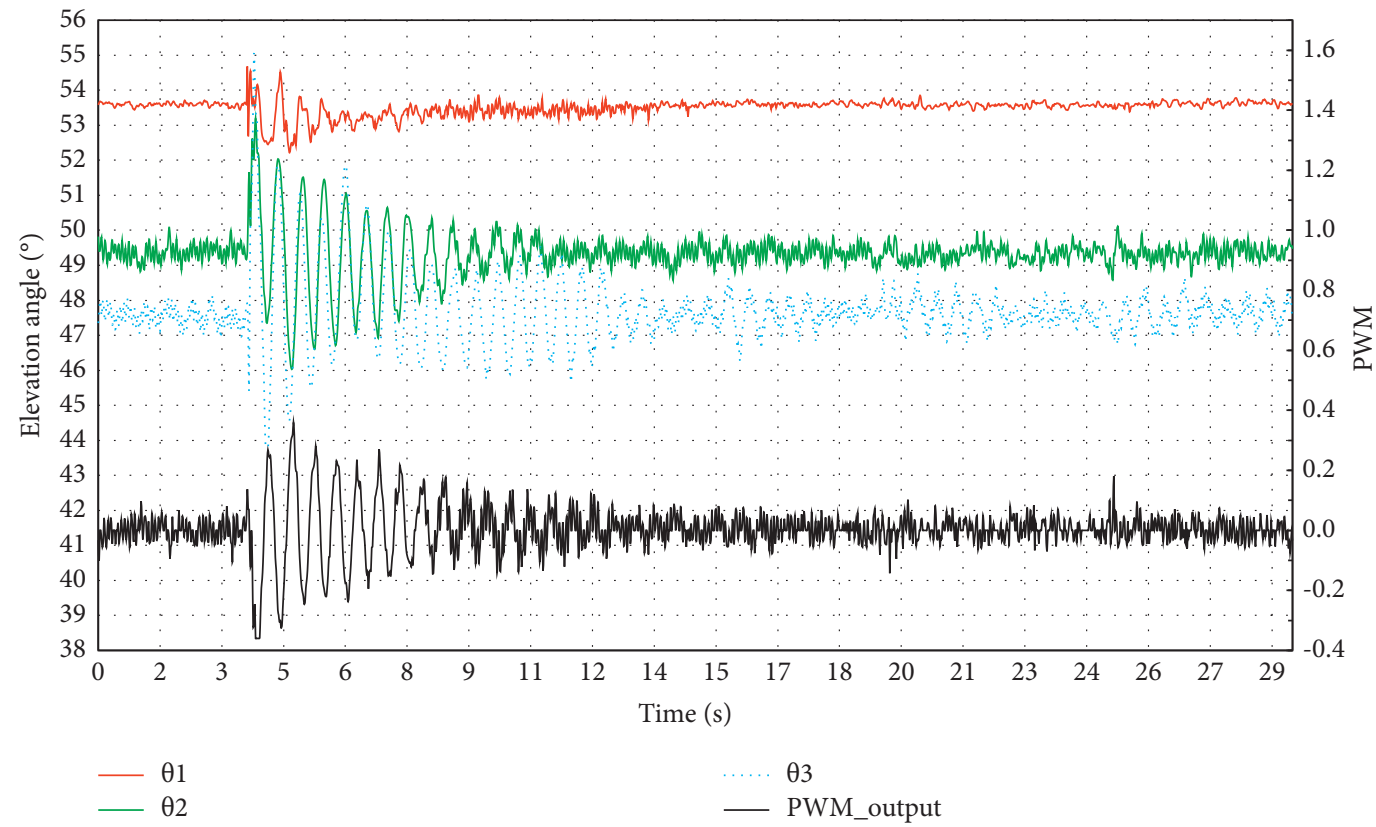

Figure 7: Vibration curve with the PID controller.

whole arm vibrates significantly under the severe impact at the initial stage, which even causes the chassis to swing. Another problem is that the effect of damping is not considered in the process of control model derivation, but the actual experimental object has its own weak distributed damping characteristics, so the LFA stabilizes slowly without any control. After being impacted, the arm needs about 90 seconds to stabilize slowly until the end vibration amplitude is less than $1^{\circ}$.

6.2.3. PID Controller Experiment. The control factors of a traditional PID controller are obtained by using the turn tool. The PID controller coefficients are as follows: proportion coefficient of 8 , differential coefficient of 4 , and integral coefficient of 0.2 . The Bode diagram of the closedloop system is shown in Figure 6. We can see that the phase margin is 69. In order to suppress the low-frequency residual vibration rapidly, the system can be stable between the first and second natural frequencies.

The PID controller with a proportion coefficient of 8 , differential coefficient of 4 , and integral coefficient of 0.2 is adopted. It can be seen from the response curve of Figure 7 that the initial impact makes the endpoint vibrate with an amplitude of about $8^{\circ}$. It takes 9 seconds for the terminal to reach the state of amplitude close to $1^{\circ}$. After that, the relatively stable stage is in the state of small-amplitude highfrequency vibration. In order to prevent more violent 


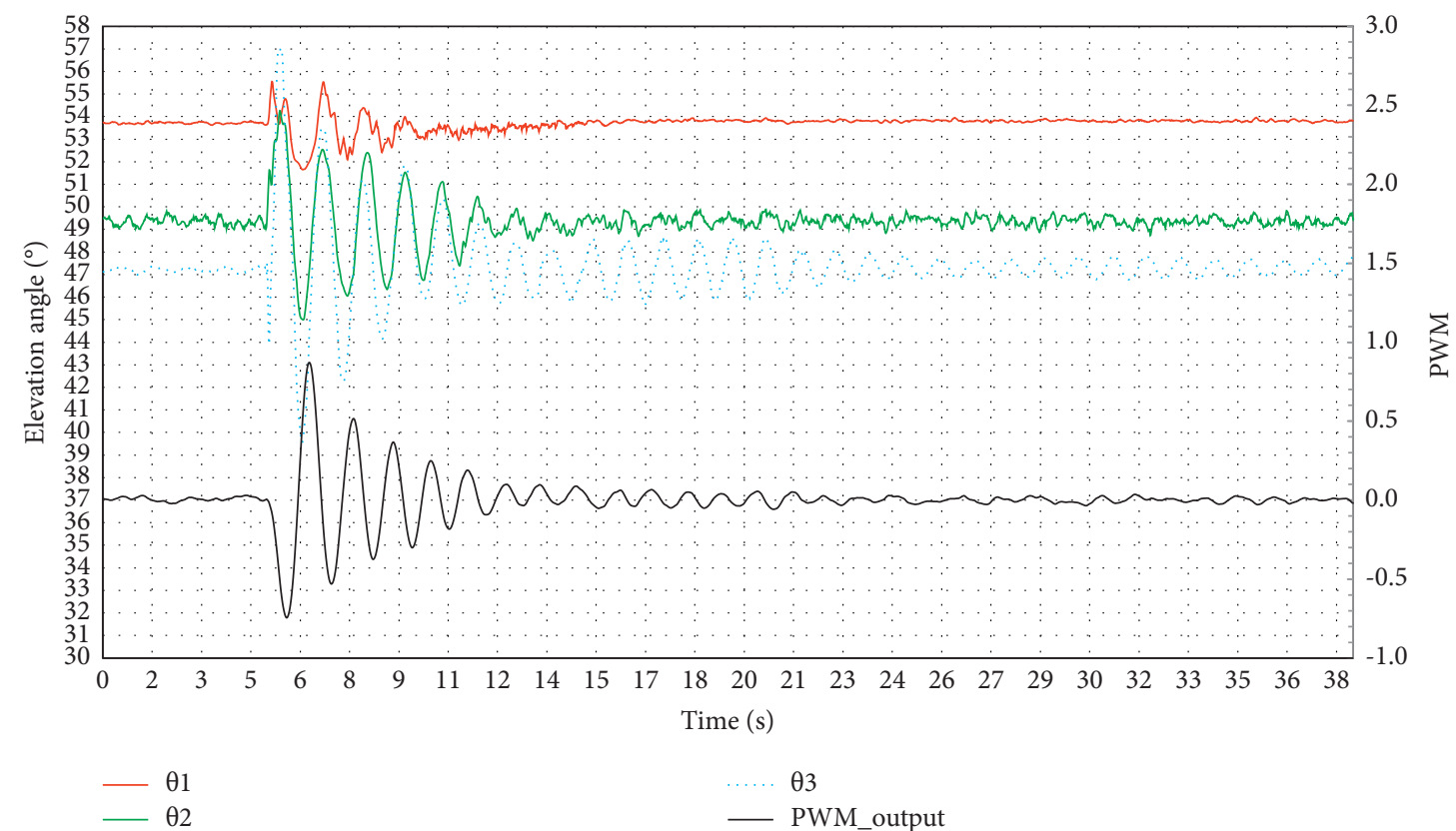

FIGURE 8: Response curve with the ADRC controller.

dynamic response, the gain used in this experiment is relatively small. The PID controller uses high-frequency response to compensate the angle deviation. Comparing Figures 5 and 6 , it is obvious that although this method can eliminate the jitter of large amplitude, the vibration frequency of small amplitude is more than twice that of free vibration.

6.2.4. ADRC Experiment. It is an attractive advantage of ADRC to substitute model information into the controller to reduce the burden of the observer. Here, eight values of the mass and length of the equivalent rigid body model and the stiffness coefficient of the equivalent torsion spring are substituted into (51), and the two important results are obtained here: $b_{o}=0.326$ and $b_{1}=-3.701$.

Take these two values into controller (60), and set the observer bandwidth as 1.1 and the controller bandwidth as 1.32. The experimental effect is shown in Figure 8. The impact load is also applied in the middle of the LFA, which makes the end jitter about $10^{\circ}$. It took six seconds for the control system to reduce the amplitude to less than $3^{\circ}$. It is worth noting that, in this way, the records of the second and third tilt sensors show that the oscillation frequency is less than $1 \mathrm{~Hz}$. After 9 seconds, the amplitude dropped to less than $1^{\circ}$. The duty cycle of the controller output is close to 0.9 , which shows that the torque capacity of the motor is utilized well.

\section{Conclusion}

It is simple and effective to establish a multibody dynamic model to characterize the LFA by means of statics and mode equivalence. The linear independence method is used to obtain the differential flat output from the three outputs, which makes the system a SISO structure. The controller based on the ADRC principle makes full use of the information of the model and skillfully uses the high-order dynamic model obtained from the differential flat output in the form of the extended state observer. The effectiveness and superiority of the method are shown in the experiment of the physical model. The control system based on the equivalent rigid body model, differential flatness, and ADRC can give full play to the ability of driving components and significantly reduce the switch frequency of the motor.

\section{Data Availability}

The data used to support the findings of this study are included within the article.

\section{Conflicts of Interest}

The authors declare no conflicts of interest.

\section{Acknowledgments}

The authors thank the Ministry of Finance and The Ministry of Agriculture, PRC, for funding this work through the Chinese Agricultural Research System (CARS-27).

\section{References}

[1] F. Raouf, M. Al-Shabi, M. Bettayeb, and J. Ghommam, "Robust active disturbance rejection control for flexible link manipulator," Robotica, vol. 38, no. 1, pp. 118-135, 2019.

[2] A. Zuyev and O. Sawodny, "Stabilization of a flexible manipulator model with passive joints," IFAC Proceedings Volumes, vol. 38, no. 1, pp. 784-789, 2005.

[3] Z.-C. Qiu and W.-Z. Zhang, "Trajectory planning and diagonal recurrent neural network vibration control of a flexible 
manipulator using structural light sensor," Mechanical Systems and Signal Processing, vol. 132, no. 1, pp. 563-594, 2019.

[4] J. Li and H. Deng, "Vibration suppression of rotating long flexible mechanical arms based on harmonic input signals," Journal of Sound and Vibration, vol. 436, pp. 253-261, 2018.

[5] R. Weldegiorgis, P. Krishna, and K. V. Gangadharan, "Vibration control of smart cantilever beam using strain rate feedback," Procedia Materials Science, vol. 5, pp. 113-122, 2014.

[6] C. Sun, W. He, and J. Hong, "Neural network control of a flexible robotic manipulator using the lumped spring-mass model," IEEE Transactions on Systems, Man, and Cybernetics: Systems, vol. 47, no. 8, pp. 1863-1874, 2017.

[7] W. He, X. He, M. Zou, and L. Hongyi, "PDE model-based boundary control design for a flexible robotic manipulator with input backlash," IEEE Transactions on Control Systems Technology, vol. 27, no. 2, pp. 790-797, 2019.

[8] L. L. Howell and A. Midha, "Parametric deflection approximations for end-loaded, large-deflection beams in compliant mechanisms," Journal of Mechanical Design, vol. 117, no. 1, pp. 156-165, 1995.

[9] A. Midha, T. W. Norton, and L. L. Howell, "On the nomenclature, classification, and abstractions of compliant mechanisms," Journal of Mechanical Design, vol. 116, no. 1, pp. 270-279, 1994.

[10] Y.-Q. Yu, Q. Li, and Q.-P. Xu, "Pseudo-rigid-body dynamic modeling and analysis of compliant mechanisms," Proceedings of the Institution of Mechanical Engineers, Part C: Journal of Mechanical Engineering Science, vol. 232, no. 9, pp. 16651678, 2018.

[11] M. Ramírez-Neria, H. Sira-Ramírez, R. Garrido-Moctezuma, and A. Luviano-Juárez, "Linear active disturbance rejection control of underactuated systems: the case of the furuta pendulum," ISA Transactions, vol. 53, no. 4, pp. 920-928, 2014.

[12] M. Ramirez-Neria, H. Sira-Ramirez, R. Garrido-Moctezuma, and A. Luviano-Juarez, "Active disturbance rejection control of singular differentially flat systems," in Proceedings of the Society of Instrument and Control Engineers Annual Conference 2015, pp. 554-559, Hangzhou, China, July 2015.

[13] R. Morales, H. Sira-Ramírez, and J. A. Somolinos, "Robust control of underactuated wheeled mobile manipulators using GPI disturbance observers," Multibody System Dynamics, vol. 32, no. 4, pp. 511-533, 2014.

[14] H. Sira-Ramírez, Active Disturbance Rejection Control of Dynamic Systems, Elsevier, Amsterdam, Netherlands, 2017.

[15] W. Cui, W. Tan, D. Li, Y. Wang, and S. Wang, "A relay feedback method for the tuning of linear active disturbance rejection controllers," IEEE Access, vol. 8, pp. 4542-4550, 2020.

[16] Z. Shen and Z. Gao, "An active disturbance rejection based approach to vibration suppression in two-inertia systems," Asian Journal of Control, vol. 15, no. 2, pp. 350-362, 2013.

[17] G. Tian and Z. Gao, "Frequency response analysis of active disturbance rejection based control system," in Proceedings of the 2007 IEEE International Conference on Control Applications, pp. 1595-1599, Singapore, October 2007.

[18] Y. Huangfu, Q. Li, L. Xu, R. Ma, and F. Gao, "Extended state observer based flatness control for fuel cell output series interleaved boost converter," IEEE Transactions on Industry Applications, vol. 55, no. 6, pp. 6427-6437, 2019.

[19] H. Sira-Ramirez and S. Agrawal, Differentially Flat Systems, Marcel Dekker, New York, NY, USA, 2004.
[20] J. Han, "From PID to active disturbance rejection control," IEEE Transactions on Industrial Electronics, vol. 56, no. 3, pp. 900-906, 2009.

[21] Z. Gao, "Active disturbance rejection control: a paradigm shift in feedback control system design," in Proceedings of the American Control Conference, pp. 2399-2405, Louis, MO, USA, June 2009.

[22] Z. Gao, "Scaling and bandwidth-parameterization based controller tuning," in Proceedings of the 2003 American Control Conference, pp. 4989-4996, IEEE Press, Denver, CO, USA, June 2003.

[23] Z. Gao, "Active disturbance rejection control: a paradigm shift in feedback control system design," in Proceedings of the American Control Conference, pp. 2399-2405, Minneapolis, MN, USA, June 2006.

[24] C. Fu and W. Tan, "Tuning of linear ADRC with known plant information," ISA Transactions, vol. 65, pp. 384-393, 2016.

[25] H. Zhang, Z. Shen, and Z. Gao, "An active disturbance rejection control solution for the two-mass-spring benchmark problem," in Proceedings of the American Control Conference IEEE, pp. 1566-1571, Boston, MA, USA, July 2016.

[26] S. S. Rao, Vibration of Continuous Systems, Wiley, Hoboken, NJ, USA, 2007. 